\title{
Vertebrobasilar artery dolichoectasia manifesting as Millard-Gubler syndrome in a young acute ischemic stroke
}

\begin{abstract}
Introduction: Vertebrobasilar dolichoectasia (VBD) is an arterial disease characterized by dilatation, elongation, and tortuosity of the vertebral and basilar arteries. VBD can generate intracranial hemorrhage, compression of the brainstem, and ischemic stroke as the most common presentation. Millard-Gubler syndrome (MGS) is a pontine-crossed syndrome affecting the pons at the level of the facial nerve nucleus. We present an unusual case of posterior circulation ischemic stroke presenting as MGS due to VBD.
\end{abstract}

Observation: A 50-year-old man, with a medical history of high blood pressure, was hospitalized after 20 hours of transient dizziness, left facial numbness, and diplopia. On examination, he had a right hemiparesis with right Babinski. Deep tendon reflexes were present. Cranial nerves examination showed left abducens paresis with left peripheral facial nerve palsy. Cranial computed tomography (CT) imaging showed left pontine infarct with dilated basilar artery. Brain MRI showed a tortuous and dilated vertebrobasilar system touching the left acoustico-facial complex nerves with a high signal intensity involving the left anterolateral pons with a small right cerebellar hemorrhage. Paraclinical tests showed high blood total cholesterol and bilateral carotid artery atherosclerosis without stenosis. The patient received aspirin, atorvastatin with anti-hypertensive agents. Two weeks after his admission, there was a resolution of the left abducens nerve paresis with mild residual left peripheral facial paresis and left hemiparesis.

Discussion: VBD is a complex arteriopathy with unknown etiology. Clinical symptoms in patients with VBD are various including brainstem and cranial nerve compression, intracranial hemorrhage, and ischemic stroke. MGS is an uncommon pontine-crossed syndrome occurring due to a lesion in the pons at the level of the facial nerve nucleus. MGS can be caused by brainstem tumor, cavernous angioma, neurocysticercosis, and cerebral infarction. The coexistence of MGS and VBD is rarer.

Conclusion: Our case provides evidence that VBD may be presented as MGS secondary to a posterior circulation ischemic stroke. Therapeutic management of patients with VBD requires a careful balancing of the benefits and risks.

Keywords: vertebrobasilar dolichoectasia, millard-gubler syndrome, ischemic stroke, antiplatelet agents
Volume 12 Issue I - 2022

\author{
M Hamid,' 'Y Adraoui,' N Mouloudi, 'Y \\ Benmoh,' A Bakkal,' A Satte, ${ }^{2}$ A Bourazza' \\ 'Department of neurology, MohamedV Military Teaching \\ Hospital, MohamedV University, Rabat, Morocco \\ ${ }^{2}$ Department of neurophysiology, MohamedV Military Teaching \\ Hospital, MohamedV University, Rabat, Morocco
}

Correspondence: Mohamed Hamid, Department of Neurology, MohamedV Military Teaching Hospital, MohamedV University, Rabat, Morocco, Tel +212 6669700 78, Email hamidsim19@gmail.com

Received: December 23, 202 I | Published: January 27, 2022

\section{Introduction}

Vertebrobasilar dolichoectasia (VBD) is a complex arteriopathy characterized by dilatation, elongation, and tortuosity of the vertebral and basilar arteries. ${ }^{1-3}$ The incidence of intracranial dolichoectasia is estimated between 0.06 and $5.8 \%$ of the population and is mostly present in people over 50 years old. ${ }^{4,5}$ The diagnostic criteria of VBD varied over literature. However, the VBD is defined if the diameter of vertebral or basilar artery $>4.5 \mathrm{~mm}$ and the length of intracranial vertebral artery $>23.5 \mathrm{~mm}$ or the length of basilar artery $>29.5 \mathrm{~mm} .{ }^{6,7}$ Clinical expressions of VBD are variable including intracranial hemorrhage, compression of the brainstem or cranial nerves, and ischemic stroke as the most common presentation. ${ }^{3,5}$

Millard-Gubler syndrome (MGS) is a pontine-crossed syndrome characterized by paralysis affecting the abducens nerve and the face on the side of the lesion and central hemiplegia on the opposite side. ${ }^{8}$ This is the first Moroccan report on the coexistence of MGS and VBD in a young acute ischemic stroke. We describe an unusual case of posterior circulation ischemic stroke presenting as MGS due to VBD.

\section{Clinical case}

A 50-year-old man was admitted 20 hours after sudden transient dizziness, right facial numbness, and diplopia. He had a medical history of hypertension treated by enalapril stopped two years ago with no other vascular risk factor or family history. On admission, the patient was conscious, well oriented, and apyretic. Neurologic examination showed left hemiparesis quoted 3/5 on Medical Research Council grading scale with left muscular hypotonia. Deep tendon reflexes were present with a left Babinski sign there was no sensory deficit and motor coordination was normal. Cranial nerves examination revealed right abducens paresis with right peripheral facial nerve palsy with no other cranial nerve involvement. Examination of other extra neurological systems was unremarkable. Clinical presentation was compatible with MGS. Cranial computed tomography (CT) imaging showed a large hyperdense focus in the right cerebellopontine angle with a dilated basilar artery (Figure 1).

Brain MRI demonstrated high signal intensity on diffusion and Flair sequences involving the right anterolateral pons referring to an 
ischemic lesion (Figure 2). The gradient-echo T2 weighted sequence showed decreased signal intensity lesion in the right cerebellar hemisphere compatible with cerebellar hemorrhage (Figure 3). T1 weighted coronal gadolinium-enhanced MRI scan showed a tortuous and dilated vertebrobasilar system (Figure 4).

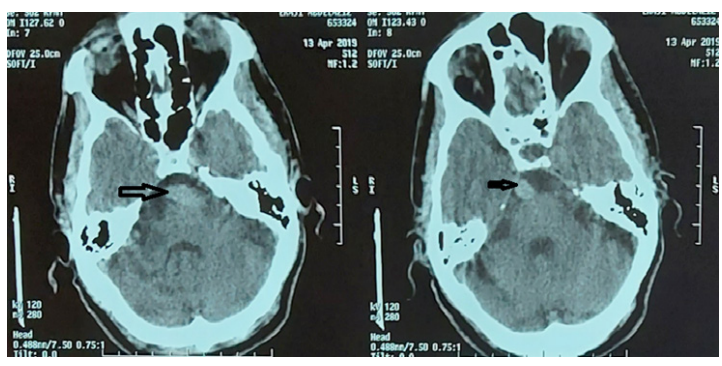

Figure I CT scan reveals a large hyperdense focus in the right cerebellopontine angle, consistent with thrombus within a dilated basilar artery (arrows).

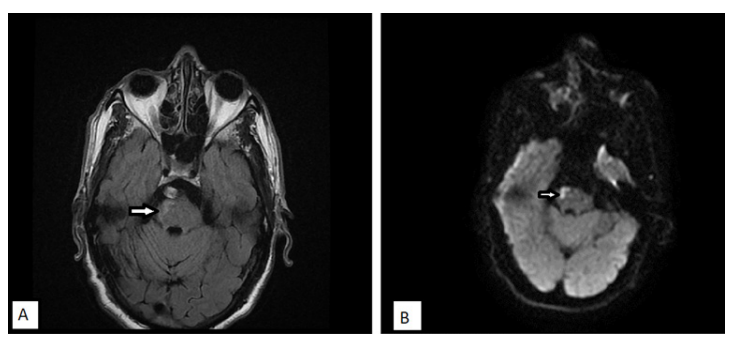

Figure 2 Cranial MRI on Flair (A) and DWI (B) sequences showed high signa intensity interesting the right anterolateral pons (white arrows).

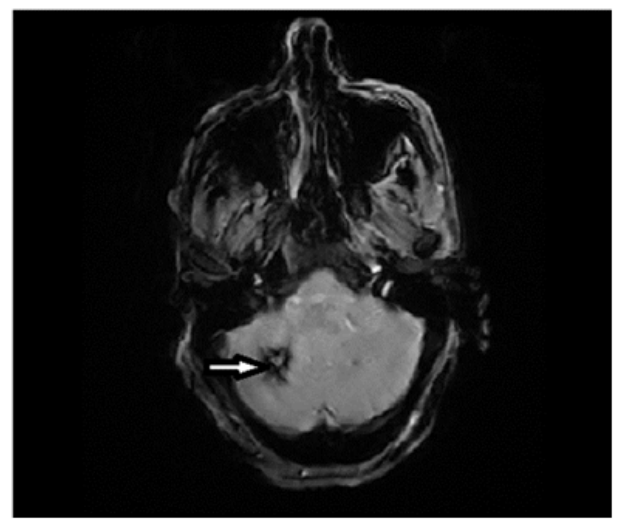

Figure 3 Axial gradient-echo T2 weighted image demonstrated decreased signal intensity lesion in the right anteroinferior cerebellar hemisphere (white arrow).

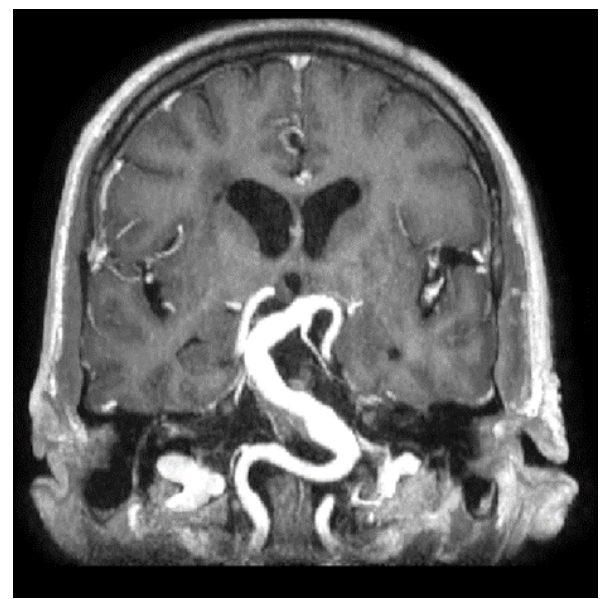

Figure 4 TI-weighted coronal gadolinium-enhanced MRI scan showing tortuous and dilated vertebrobasilar system with diameter $>8 \mathrm{~mm}$.
Paraclinical tests were normal (complete blood count, glycosylated hemoglobin, renal and hepatic tests). Blood total cholesterol was elevated $(7.32 \mathrm{mmol} / \mathrm{L})$ with normal low-density lipoprotein (1.3 $\mathrm{mmol} / \mathrm{L}$ ). Electrocardiogram and transthoracic echocardiography were normal. Doppler ultrasound of cervical arteries showed bilateral carotid artery atherosclerosis without stenosis. The patient was treated with aspirin $(160 \mathrm{mg})$ and atorvastatin $(20 \mathrm{mg})$ with strict blood pressure control by anti-hypertensive agents, and he underwent functional rehabilitation two weeks after his admission, examination showed resolution of the left abducens nerve paresis with mild residual left peripheral facial paresis and left hemiparesis.

\section{Discussion}

The pathophysiology of vertebrobasilar dolichoectasia (VBD) has been extensively studied, the etiology and the factors that promote its development are still unclear. Atherosclerosis was considered the main cause of intracranial dolichoectasia because the disease tends to occur in older people with hyperlipidemia and hypertension. ${ }^{9}$ Histological findings in atherosclerotic lesions demonstrated typically thickening of the intima. ${ }^{10}$ However, microscopic features of dolichoectatic arteries include fragmentation of the internal elastic lamina and thinning of the tunica media, which are different from those seen in atherosclerosis. ${ }^{10-12}$

The involvement of both hereditary and acquired factors in VBD is recently discussed. ${ }^{10}$ Hereditary factors including Pompe disease, autosomal dominant polycystic kidney disease, Fabry's disease, and Marfan's syndrome have been related to VBD $(3,10,13$ 15). The development of VBD can be caused by some infections such as varicella-zoster, syphilis, and human immunodeficiency virus infection. ${ }^{16-18}$ Whatever the cause, it is now believed that the development of VBD progress over time, with hypertension as a possible factor in the development of neovascularization and intimal hyperplasia followed by intramural hemorrhage and thrombosis. ${ }^{19}$

Clinical symptoms in patients with VBD are various, but the main clinical presentations are brainstem and cranial nerve compression, intracranial hemorrhage, and ischemic stroke. In the prospective study of Passero and colleagues ${ }^{20} 37.8 \%$ of 156 patients reported ischemic stroke, $19.9 \%$ had brainstem and cranial nerve brainstem compression, and $13.5 \%$ of patients had intracranial hemorrhage. The cerebral infarcts were in the brainstem (41\%), followed by the posterior cerebral artery territory (29\%), and cerebellum (2\%).

Ischemic stroke is usually occurring due to intravascular thrombus formation in the dolichoectatic artery caused by the alteration in blood flow (15). When the basilar artery becomes increasingly tortuous and elongated, the paramedian perforating arteries supplying the pons become distorted and occluded, leading to pontine infarction., ${ }^{1,21}$ Our patient had a clinical presentation of MGS which is unusual as a pontine-crossed syndrome and as a clinical feature of VBD. MGS is occurring due to a lesion in the pons at the level of the facial nerve nucleus. In this case report, the ischemic lesion was found in the left anteromedial pons at the level of the facial nerve nucleus crossing the abducens nerve and the corticospinal tract, due to occlusion of the left paramedian perforating arteries caused by the thrombus in the basilar dolichoectasia.

It has been reported that MGS can be caused by brainstem tumors, cavernous angioma, and neurocysticercosis. ${ }^{22-24}$ Only a few cases of MGS secondary to cerebral infarction have been described in the literature. ${ }^{25-28}$ As far as we know, the coexistence of MGS and VBD has not been reported yet. 
Given the lack of evidence-based clinical guidelines for the management of ischemic stroke caused by VBD, the treatment of patients with VBD remains a challenge. Anticoagulants and antiplatelet agents have been used if an intravascular thrombus is found. 3,29

Small studies confirmed that there is no difference in outcome between treatment with anticoagulants and antiplatelet agents in patients with VBD. ${ }^{30}$ However, patients with VBD treated by antiplatelet or anticoagulant medications with poorly controlled hypertension showed a high risk of cerebral hemorrhage (29).

The patient in this report has a pontine infarction with small cerebellar hemorrhage treated by antiplatelet agents with strict blood pressure control by anti-hypertensive agents with a favorable outcome. Invasive treatment by coil-assisted stent reconstruction or overlapping stent has been newly reported necessitating experienced neurosurgical operators, with varying degrees of success. ${ }^{31-33}$

\section{Conclusion}

Vertebrobasilar dolichoectasia (VBD) is a progressive arterial disease with variable clinical features, including brainstem and cranial nerve compression, intracranial hemorrhage, and especially ischemic stroke. we reported an unprecedented case of coexistence of VBD and MGS in an ischemic stroke patient. The therapeutic approach of patients with VBD requires a careful balancing of the benefits and risks, with the need for more research and studies on the management of intracranial dolichoectasia.

\section{Acknowledgments}

None.

\section{Conflicts of interest}

The authors declare having no conflict of interest.

\section{References}

1. Nishizaki T, Tamaki N, Takeda N, et al. Dolichoectatic basilar artery:A review of 23 cases. Stroke. 1986;17:1277-1281.

2. Sarikaya S, Sarikaya B. Natural history of vertebrobasilar dolichoectasia. Neurology. 2008;71(18):1460.

3. Yuan YJ, Xu K, Luo Q, et al. Research progress on vertebrobasilar dolichoectasia. Int J Med Sci. 2014;11:1039-1048.

4. Yu YL, Moseley IF, Pullicino P, et al. The clinical picture of ectasia of the intracerebral arteries. J Neurol Neurosurg Psychiatry. 1982;45:29-36.

5. Flemming KD, Wiebers DO, Brown RD Jr, et al. The natural history of radiographically defined vertebrobasilar nonsaccular intracranial aneurysms. Cerebrovasc Dis. 2005;20:270-279.

6. Smoker WR, Price MJ, Keyes WD, et al. High-resolution computed tomography of the basilar artery: 1 . Normal size and position. Am $J$ Neurol Radiol. 1986;7:55-60.

7. Smoker WR, Corbett JJ, Gentry LR, et al. High-resolution computed tomography of the basilar artery: 2. Vertebroba silardo -lichoectasia:clinical-pathologic correlation and review. Am J Neurol Radiol. 1986;7:61-72.

8. Onbas O, Kantarci M, Alper F, et al. Millard-Gubler syndrome:MR findings. Neuroradiology. 2005;47:35-37.

9. Dziewasa R, Freund M, Ludemann P, et al. Treatment options in vertebrobasilar dolichoectasia - case report and review of the literature. Eur Neurol. 2003;49(4):245-247.
10. Lou M, Caplan LR. Vertebrobasilar dilatative arteriopathy (dolichoectasia). Ann NY Acad Sci. 2010;1184:121-33.

11. Anson JA, Lawton MT, Spetzler RF. Characteristics and surgical treatment of dolichoectatic and fusiform aneurysms. J Neurosurg. 1996;84:185-193.

12. Zis $\mathrm{P}$, Fragkis $\mathrm{S}$, Lykouri M, et al. From basilar artery dolichoectasia to basilar artery aneurysm:natural history in images. $J$ Stroke Cerebrovasc Dis. 2015;24:e117-e19.

13. Schievink WI, Torres VE, Wiebers DO, et al. Intracranial arterial dolichoectasia in autosomal dominant polycystic kidney disease. $J \mathrm{Am}$ Soc Nephrol. 1997;8:1298-1303.

14. Pico F, Labreuche J, Seilhean D, et al. Association of small-vessel disease with dilatative arteriopathy of the brain:neuropathologic evidence. Stroke. 2007;38(4):1197-202.

15. Caplan LR. Dilatative arteriopathy (dolichoectasia): What is known and not known. Ann Neurol. 2005;57:469-471.

16. Lodder J, Vles JS, Broekman JM. Megadolicho anomaly of the basilar artery caused by syphilis (a case report). Clin Neurol Neurosurg. 1982;84(4):255-259.

17. Dalton CM, Jager HR, Losseff NA, et al. Varicella zoster virus and intracranial dolichoectasia in a late adult cancer survivor. BMJ Case Rep. 2009;2009.

18. Mahadevan A, Tagore R, Siddappa NB, et al. Giant serpentine aneurysm of vertebrobasilar artery mimicking dolichoectasia - an unusual complication of pediatric AIDS. Report of a case with review of the literature. Clin Neuropathol. 2008;27:37-52.

19. Nakatomi H, Segawa H, Kurata A et al. Clinicopathological study of intracranial fusiform and dolichoectatic aneurysms: Insight on the mechanism of growth. Stroke. 2000;31:896-900.

20. Passero SG, Rossi S. Natural history of vertebrobasilar dolichoectasia. Neurology. 2008;70:66-72.

21. Passero S, Filosomi G. Posterior circulation infarcts in patients with vertebrobasilar dolichoectasia. Stroke. 1998;29:653-594.

22. Kuhn J, Brümmendorf TH, Brassat U, et al. Novel KRIT1 mutation and no molecular evidence of anticipation in a family with cerebral and spinal cavernous malformations. Eur Neurol. 2009;61:154-158.

23. Ben Nsir A, Badri M, Kassar AZ, et al. Hemangiopericytoma of the Cerebellopontine Angle:A Wolf in Sheep's Clothing. Brain Tumor Res Treat. 2016;4:8-12.

24. Prasad R, Kapoor K, Srivastava A, et al. Neurocysticercosis presenting as Millard Gubler syndrome. J Neurosci Rural Pract. 2012;3(3):375-377.

25. Ahdab R, Saade HS, Kikano R, et al. Pure ipsilateral central facial palsy and contralateral hemiparesis secondary to ventro-medial medullary stroke. J Neurol Sci. 2013;332:154-155.

26. Matlis A, Kleinman Y, Korn-Lubetzki I. Millard-Gubler syndrome. AJNR Am J Neuroradiol. 1994;15:179-181.

27. Yasuda Y, Matsuda I, Sakagami T, et al. Pontine infarction with pure Millard-Gubler syndrome:precise localization with magnetic resonance imaging. Eur Neurol. 1993;33:331-334.

28. Rose DZ, Parra-Herran C, Petito CK, et al. Restricted Diffusion of Pus in the Subarachnoid Space:MRSA Meningo-Vasculitis and Progressive Brainstem Ischemic Strokes - A Case Report. Case Rep Neurol. 2010;2:101-110.

29. Passero SG, Calchetti B, Bartalini S. Intracranial bleeding in patients with vertebrobasilar dolichoectasia. Stroke. 2005;36:1421-1425.

30. Rabb CH, Barnwell SL. Catastrophic subarachnoid hemorrhage resulting from ruptured vertebrobasilar dolichoectasia: Case report. Neurosurgery. 1998;42(2):379-382. 
31. Cohen JE, Gomori JM, Moscovici S, et al. Successful endovascular treatment of a growing megadolichoectasic vertebrobasilar artery aneurysm by flow diversion using the "diverter-in-stent" technique. J Clin Neurosci. 2012;19(1):166-170.

32. Chen Z, Yang $\mathrm{Y}$, Miao $\mathrm{H}$ et al. Endovascular treatment for large and giant fusiform aneurysms of the vertebrobasilar arteries. Clin Imaging. 2013;37:227-231.
33. Siddiqui AH, Abla AA, Kan P, et al. Panacea or problem:Flow diverters in the treatment of symptomatic large or giant fusiform vertebrobasilar aneurysms. J Neurosurg. 2012;116:1258-1266. 